\title{
Therapeutic Effect of Propranolol in Mexican Patients with Infantile Hemangioma
}

\author{
Saul Castaneda ${ }^{1,3}$ (ID Esbeydy Garcia ${ }^{2} \cdot$ Hermelinda De la Cruz ${ }^{1,3}$. \\ Oscar Ramirez ${ }^{3} \cdot$ Samuel Melendez $^{3} \cdot$ Jose Sanchez-Palacio $^{3}$
}

Published online: 21 December 2015

(c) The Author(s) 2015. This article is published with open access at Springerlink.com

\begin{abstract}
Background Infantile hemangiomas are the most common childhood vascular tumors, occurring in $10 \%$ of children aged less than 1 year. Propranolol, a $\beta$-adrenergic blocker mainly indicated for hypertension, has proven effective in treating these types of tumors.

Objective To evaluate the efficacy and adverse effects of propranolol in Mexican pediatric patients diagnosed with infantile hemangioma, treated with an extemporaneously compounded solution of propranolol.

Methods An open prospective observational study at the Children's Hospital of the Californias in Tijuana, Mexico was performed on ambulatory pediatric patients between the ages of 3 and 12 months diagnosed with infantile hemangioma. Patients were treated with an oral solution of propranolol in doses ranging from 0.5 to $2.5 \mathrm{mg} / \mathrm{kg} /$ day. Children were monitored monthly by the physician in charge, at which time clinical and treatment data were collected.

Results Over a period of 20 months, 31 patients were treated (36\% male and $64 \%$ female).The majority of hemangiomas were superficial (55\%), located mainly on
\end{abstract}

Saul Castaneda

saulcashe@gmail.com

1 Department of Pharmacy, Children's Hospital of the Californias, Av. Alejandro von Humboldt 11431 y Garita de Otay, 22509 Tijuana, Baja California, Mexico

2 Department of Pediatric Dermatology, Children's Hospital of the Californias, Tijuana, Baja California, Mexico

3 Faculty of Chemical Sciences and Engineering, Autonomous University of Baja California, Tijuana, Mexico the face. Treatment had an average duration of 10.5 months. Ninety-six percent responded to the treatment, showing decreases in size and coloration of the hemangioma. Children who started therapy before 5 months of age had a significantly better response and shorter duration of treatment. The average therapeutic dose was $1.5 \mathrm{mg} / \mathrm{kg} / \mathrm{day}$. Five patients experienced mild adverse effects during the first month of therapy.

Conclusion Treatment with propranolol in this group of Mexican pediatric patients proved to be safe and effective at an average dose of $1.5 \mathrm{mg} / \mathrm{kg} / \mathrm{day}$, reducing the size and coloration of hemangioma with a minimum incidence of adverse effects.

\section{Key Points}

Propranolol is the first line treatment for infantile hemangiomas, showing good results in the Mexican population.

Use of an extemporaneously compounded solution of propranolol in this study proved to be an effective and lowcost alternative to commercially available proprietary formulations.

Children with hemangioma who started therapy with propranolol before five months of age had a significantly better response and shorter duration of treatment.

Success of therapy was dependent on continuous medical supervision by and the pharmaceutical education of the parents, which facilitated outpatient treatment and greater therapeutic adherence. 


\section{Introduction}

Infantile hemangiomas (IH) are the most common benign vascular tumors of childhood, with an incidence of 5-10\% during the first year of life. They are more frequent in Caucasians and premature infants, and three times more frequent in female patients than in males [1]. They can affect any area of the body's surface, although in most cases they tend to be located in the head and neck area [2].

Three stages have been identified in the development of IH: initial, proliferative, and involution. The etiology of IH is still unknown; some evidence suggests that vascular endothelial growth factor (VEGF) and basic fibroblast growth factor (bFGF) are involved, as their levels increase during the development of the vascular tumor $[3,4]$.

Even though most IH are benign, self-limiting, and require no treatment, some $\mathrm{IH}$ can cause complications such as ulceration and even permanent disfigurement. Treatment may be necessary for IH during the proliferative phase when located in key organs of the body as they may endanger organ function. Standard treatments for $\mathrm{IH}$ include the use of drugs such as corticosteroids, other immunosuppressants, or $\beta$-adrenergic blockers, as well as surgery and laser pulses [5].

Propranolol is a non-selective $\beta$-adrenergic blocker indicated for hypertension in pediatric patients. Its role in the treatment of IH was discovered and first reported by Léauté-Labréze et al. [6], who demonstrated that oral administration of the drug during the growth phase of the hemangioma resulted in decreases in both its size and its coloration, without serious adverse effects. Several hypotheses regarding the pharmacodynamics of propranolol on IH have emerged: these include vasoconstriction, decreased expression of VEGFs, and induction of apoptosis, and other expression of components of the renin-angiotensin system in proliferating infantile hemangioma may account for the propranolol-induced accelerated involution [3, 4].

In Mexico, only a limited number of clinical studies have been published, most of which included only small numbers of patients [7,8], and failed to report important data such as age of onset, duration, adverse effects, etc. These omissions have prevented the adequate assessment of the effectiveness of the drug in the Mexican pediatric population presenting with the disease. Added to this is the lack of a commercial propranolol formulation adapted for the pediatric population.

The aim of this study was to evaluate the efficacy and adverse effects of propranolol administered orally to a group of Mexican pediatric patients diagnosed with infantile hemangioma treated with an extemporaneously compounded solution of propranolol.

\section{Methods}

\subsection{Settings}

An open observational prospective study was performed during the period from March 2013 to November 2014 in the Children's Hospital of the Californias in Tijuana, Mexico. Pediatric patients who were seen by dermatologists at this hospital and other hospitals in the private and public sectors and who were sent to the pharmacy of this hospital for the drug formulation were included.

\subsection{Patient Selection}

In our study, we used exclusively outpatient treatment, with follow-up by a physician and a clinical pharmacist.

Outpatients with ages ranging from 3 to 24 months of age who had been clinically diagnosed with hemangioma in the initial or proliferative phase were eligible for treatment. Indications for treatment were functional impairment, ulceration, rapid growth, and esthetic complications. All patients included in the study had no previous history of treatment with any drug or any other therapy $[9,5]$.

\subsection{Treatment Protocol}

A clinical history was taken before starting treatment, including vital signs, and physical examinations to confirm that the patients did not have any contraindications for the administration of propranolol, such as cardiopulmonary disease, respiratory tract infection, asthma, sinus bradycardia, and heart block, among others [10, 9]. Cardiologic examinations such as ECG and blood pressure measurements were performed by the cardiologist referred by the physician before the start of therapy, at baseline, and during therapy at each of the patient's scheduled visits for prescription refills, every 30 days until the end of the treatment, in order to rule out contraindications for the use of propranolol. None of the patients were hospitalized for evaluation at the beginning of the study.

The initial dose of propranolol was $0.5 \mathrm{mg} / \mathrm{kg} / \mathrm{day}$ administered every $8 \mathrm{~h}$. In the course of treatment, the dose was increased according to the patient's weight and tolerance, without exceeding $2.5 \mathrm{mg} / \mathrm{kg} / \mathrm{day}$. At the beginning, parents were informed of the possible adverse effects of the treatment and asked to report them if any appeared, and they were instructed to administer the drug with meals.

Measurements of mixed and deep IH were done with medical grade tape initially and at each follow-up in order to determine the area and volume of IH to correlate with the effect and duration of treatment. Even though the method previously reported by Berck et al. [11] is not the 
most accurate for the measurement of $\mathrm{IH}$, we decided to use it because it is a non-invasive procedure, and it gives a good approximation of the size of the IH. Photographs of the IH were taken every 30 days to evaluate the change in coloration [5].

The clinical response to propranolol was classified as either satisfactory or absent. A satisfactory response was considered when there was a greater than $80 \%$ reduction in the coloration and hemangioma size, with minimal visible lesions at the end of therapy, such as telangiectasias. It was classified as absent when the effect was slow, with little evidence of a decreasing IH.

Therefore, treatment was considered successful in this study when a satisfactory response was obtained, taking into account the measurements of hemangioma, serial photographs, and visible clinical effect, as subjectively assessed by two observers. After reaching a satisfactory therapeutic response, the dose was gradually decreased by half every 2 weeks until the therapy was completed.

The attending physicians referred the patients to the hospital pharmacy with a prescription for the preparation of extemporaneous formulations of propranolol of $1 \mathrm{mg} / \mathrm{ml}$ or $2 \mathrm{mg} / \mathrm{ml}$; the syrup was prepared using propranolol hydrochloride tablets (Inderalici ${ }^{\circledR}$ ) and United States Pharmacopeia (USP) grade sucrose in accordance with the standard procedures described in the Mexican Pharmacopoeia and literature reviews for compounding preparations, with an expiration date of 15 days [12, 13]. The extemporaneous formulation was prepared for each patient according to the dose prescribed by the doctor, and before or at the expiration date, a family member went back to refill the prescription.

\subsection{Clinical and Pharmacotherapeutic Monitoring}

Evaluation of the treatment was based on a clinical examination that included body weight, heart rate, blood pressure, measurement of the $\mathrm{IH}$, and analysis of photographs taken conducted by a team of physicians that included a dermatologist and a pediatric surgeon.

The pharmacist was responsible for preparing the medication and reminding the patient's family about its proper administration, and asking about possible adverse effects.

All demographic and clinical data (body weight, age of the patient, type of infantile hemangioma, and therapy data such as dose, duration of treatment, side effects, and therapeutic response) from the patients were recorded from the beginning to the end of treatment.

\subsection{Statistical Analysis}

Analysis was performed using the Sigma Plot 11.0 Software. All data are expressed as numbers, percentages, or means \pm standard deviations. Student's $t$ test was used for comparisons between two groups. For three groups a oneway analysis of variance was used. A statistical threshold of $p<0.05$ was used as the criterion for statistical significance.

\section{Results}

A total of 31 pediatric patients were treated with a propranolol syrup in the period from March 2013 to November 2014; 20 patients were female $(65 \%)$ and 11 were male (35\%). Patients' ages ranged between 3 and 12 months. The female/male ratio was $2: 1$.

\subsection{Types of Infantile Hemangioma (IH)}

Fifty-five percent of cases treated corresponded to superficial hemangiomas predominantly located in the face. In total there were 38 infantile hemangiomas. Five patients had more than one IH located elsewhere in the body. The main clinical data are shown in Table 1.

Table 1 Clinical data of Mexican patients with infantile hemangiomas treated with propranolol

\begin{tabular}{ll}
\hline Characteristic & $n(\%)$ \\
\hline Gender & \\
Male & $11(36)$ \\
Female & $20(64)$ \\
Type of hemangioma & \\
Surface & 17 \\
Deep & 6 \\
Mixed & 8 \\
Hemangioma localization ${ }^{\text {a }}$ & \\
Head and neck & $25(66)$ \\
Head & 3 \\
Cheek & 6 \\
Nose & 4 \\
Neck & 5 \\
Chin and lips & 4 \\
Periocular & 3 \\
Trunk & $7(18)$ \\
Back & 4 \\
Chest & 3 \\
Extremities & \\
Arms & $6(16)$ \\
Legs & 5 \\
Ulcerated hemangiomas & 1 \\
\hline
\end{tabular}

${ }^{a}$ Total of infantile hemangiomas $=38$ (some patients had more than one $\mathrm{IH}$ ) 


\subsection{Age of Onset}

Patients started treatment at an average age of 5.6 months, with a range of 3-11 months. In 17 cases (55\%), the IH was in the initial and/or in the proliferative phase.

Table 2 Data on treatment with propranolol in Mexican patients with infantile hemangiomas

\begin{tabular}{ll}
\hline Characteristic & Result \\
\hline Median age at onset, months & $5.6( \pm 2.7 \mathrm{SD})$ \\
Dose of propranolol, mean & $1.5 \mathrm{mg} / \mathrm{kg} / \mathrm{day}^{\mathrm{a}}$ \\
Median duration of treatment by location, months & 10.1 \\
& $( \pm 3.5 \mathrm{SD})$ \\
Head and neck & 9.4 \\
Trunk & 9.7 \\
Extremities & 11.8 \\
Side effects, $n=5$ & \\
Insomnia & 2 \\
Diarrhea & 2 \\
Hypotension & 1 \\
Therapeutic response, $n=31$ & 30 \\
Satisfactory & 1 \\
Absent & \\
\hline
\end{tabular}

${ }^{a}$ Dose range of $0.5-2.5 \mathrm{mg} / \mathrm{kg} / \mathrm{day}$

Fig. 1 Four-month-old boy with a segmental infantile hemangioma in the face treated with propranolol at months 0 (a), 5 (b), and 8 (c)

\subsection{Response and Duration}

The average continuous treatment had a duration of 10.5 months (range 7-14 months) in the study period (Table 2).

The response to treatment was observed in the first 15 days of titration in most cases, as a slight decrease in the coloration of the hemangioma. Satisfactory response to treatment was observed in $96 \%$ of the patients, with minimal noticeable residual lesions. The average dose was $1.5 \mathrm{mg} / \mathrm{kg} / \mathrm{day}$, with a range of $0.5-2.5 \mathrm{mg} /$ $\mathrm{kg} /$ day. The response was assessed by performing measurements of the IH and serial photography (Figs. 1, 2). The duration of treatment was variable, with treatment courses being shorter for patients with IH located in the head and neck. Therapy was completed when the patient had a satisfactory response. In six cases, after completing the propranolol therapy, topical treatments were continued with an extemporaneously compounded preparation of $0.5 \%$ timolol gel and corticosteroid such adapalene, to help remove residual lesions and telangiectasias.

One patient included in this study who had an IH located on the lower lip and face did not respond adequately to treatment in the first 6 months, or to an increase in the dosage.

A female patient with an IH located on her head presented regrowth after discontinuation of the treatment at
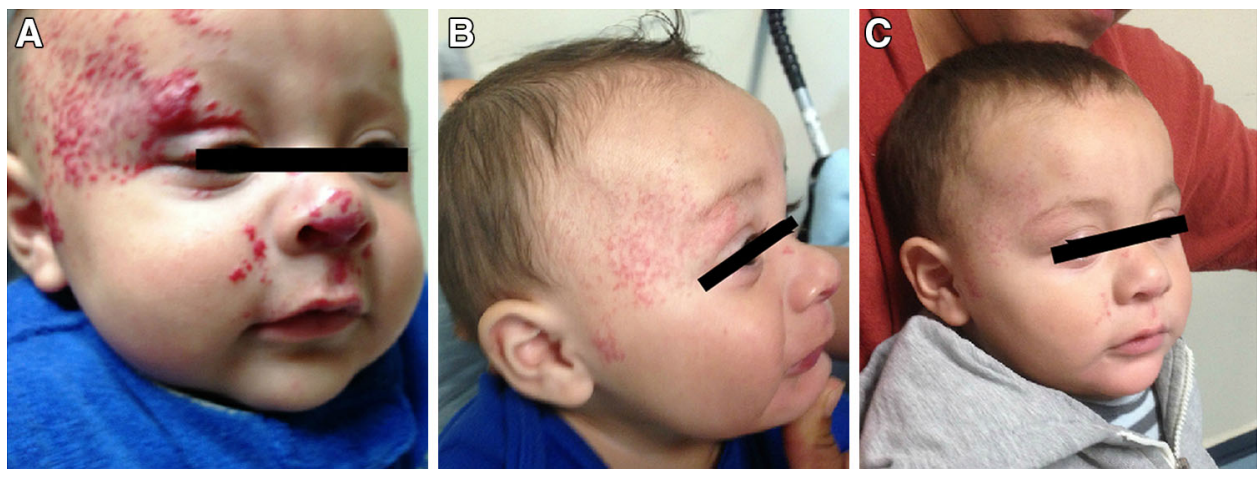

Fig. 2 Six-month-old boy with a mixed hemangioma in the superior trunk treated with propranolol at months $0(\mathbf{a}), 3$ (b), and 6 (c)
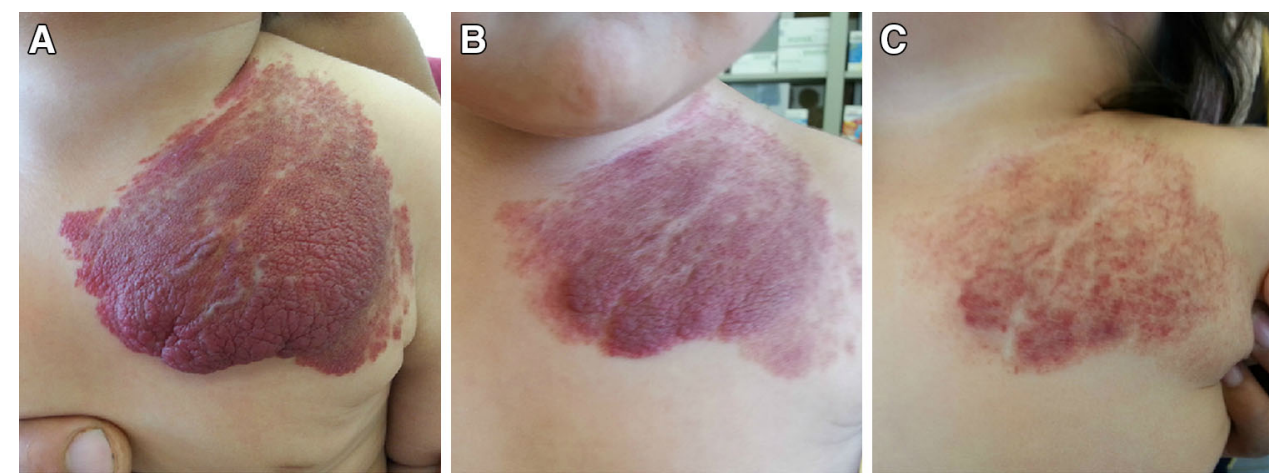
Fig. 3 Effect of propranolol in decreasing the size of deep and mixed hemangiomas from the beginning until the end of therapy

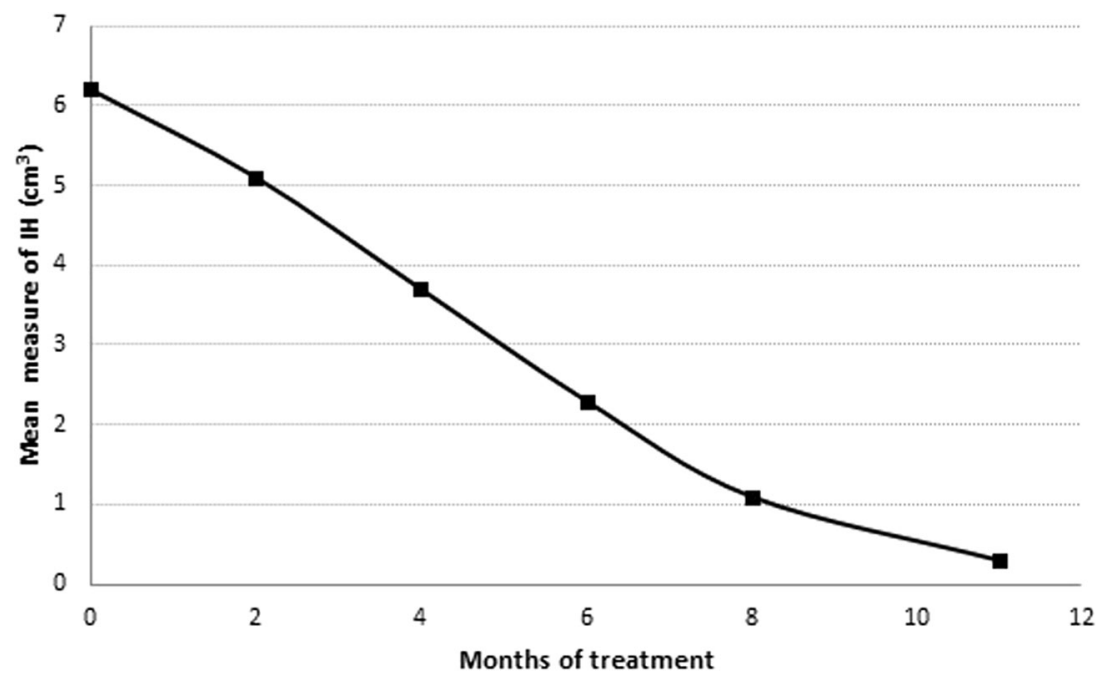

7 months, later resuming therapy with a dosage of $2 \mathrm{mg} /$ $\mathrm{kg} /$ day for 3 months until responding with total resolution of the hemangioma.

\subsection{Effect in Relation to Location Type and Onset of Treatment}

In patients with IH located in the head and neck area, a satisfactory response was reached on average in 9.4 months, whereas for patients with IH located on the trunk and/or extremities a satisfactory response was reached in an average of 10.7 months. Children who started treatment at less than 5 months of age had better responses and a shorter duration of treatment than those who started after 5 months of age. The mean durations of treatment were $8.2( \pm 1.5 \mathrm{SD})$ and $11.05( \pm 1.2 \mathrm{SD})$ months, respectively (paired Student's test, $p=0.004$ ). For the type of IH, the one-way ANOVA indicated that superficial lesions had greater duration than deep and mixed IH with a mean of $11.05( \pm 2.1 \mathrm{SD})$ and $8.6( \pm 1.1$ $\mathrm{SD})$ months, respectively $(p=0.025)$.

\subsection{Measurements of the IH}

For deep and mixed $\mathrm{IH}$, measurements were obtained at the beginning of and during treatment. On average, every IH measured about $6.5 \mathrm{~cm}^{3}$ at the beginning. A mean reduction of $16 \%$ was observed in the first 2 months of treatment, $50 \%$ or greater in the fifth month, and almost $100 \%$ after 10 months in this type of IH (Fig. 3). Measurements taken in the course of our study showed that deep and mixed IH were reduced an average of $10 \%$ of their total size every month during a 10 -month treatment.

\subsection{Adverse Effects}

During the study only five patients (16\%) presented mild adverse effects, which were self-limiting. Two patients presented with sleep disorders and two developed diarrhea. In these cases propranolol was suspended for a week, the dosage was reduced by half for each of them, and then gradually increased as tolerated by patient, until the therapy was completed. One patient presented with mild hypotension $(75 / 55 \mathrm{mmHg})$ [9]. The effects occurred during the first 6 weeks of treatment, especially when the dosage was increased. No patient discontinued therapy due to adverse effects.

\section{Discussion}

Propranolol is a nonselective $\beta$-adrenergic blocking agent. It has been used for over 50 years in pediatric and adult patients for the treatment of hypertension, heart failure, tachycardia, and acute myocardial infarction. The doses used in pediatric patients range from 0.5 to $6 \mathrm{mg} / \mathrm{kg} / \mathrm{day}$ [14]. After the discovery of the effect of propranolol on IH, numerous studies around the world have been published, describing satisfactory responses in pediatric populations [5, 15-20], with a higher cure rate and fewer adverse effects than corticosteroids, formerly the first choice of treatment for this condition [21].

So far, in Mexico only two studies have been published since this discovery. Gonzalez et al. [7] observed the effect of propranolol in five patients with periorbital $\mathrm{IH}$ who obtained good results. Orozco-Covarrubias et al. [8] reported only the demographic and clinical characteristics of 26 patients with IH treated with propranolol, but did not 
report any clinical results of treatment. The clinical and demographic characteristics of our patients are consistent with those described previously in the literature, with a predominance of females and location in the head and neck $[1,2,8]$. The range of ages at which children in this study began treatment was 3-11 months, at a time when the IH was in its initial and/or growing phase.

Several initial doses of propranolol have been reported for the treatment of $\mathrm{IH}$, for example the American Consensus Conference has suggested an initial dosage of $1 \mathrm{mg} /$ $\mathrm{kg} /$ day followed by a gradual dosage escalation to $2 \mathrm{mg} /$ $\mathrm{kg} / \mathrm{day}$ [9]. Other studies have reported the use of $2-3 \mathrm{mg} /$ $\mathrm{kg} / \mathrm{day}[17,20]$ and $0.75-1 \mathrm{mg} / \mathrm{kg} /$ day [18]. Larger scale studies confirming the safety and efficacy of propranolol may broaden the indications of treatment of proliferating $\mathrm{IH}$ [22]. Since there is no established dosage in the population, we considered a range of $0.5-2.5 \mathrm{mg} / \mathrm{kg} /$ day to be appropriate for and tolerated by our population. The extemporaneous formulation prepared with USP-grade sucrose and flavor facilitated the acceptance of the medication without potentially harmful preservatives $[12,13]$.

In $96 \%$ of the cases, the response to propranolol was satisfactory. Statistical results show that children who started treatment before 6 months of age had a significantly better response than those who started later. Similar results have been reported by Andersen et al. [23]. IH proliferates rapidly in the first 3-6 months after birth, which may explain why the effect of treatment is most evident at this time. The early initiation of therapy appeared to help prevent longer treatment protocols.

The duration of therapy was adjusted for each type of hemangioma and treatment indication. The average age at which therapy was initiated and completed was 5.6 and 16.1 months, respectively. With regard to the location, patients with head and neck hemangioma needed on average a shorter time of therapy of about 9.4 months. Most published studies have been done in European populations [5, 16, 20, 21, 24]. In these studies the average duration of therapy was 7.2 months, which is lower than that reported in our study, i.e. 10.5 months. Based on these studies the physicians decided to lengthen the treatment to over 8 months to prevent possible regrowth.

Notably, in most cases the use of propranolol led to an almost complete reduction in the size and coloration of the $\mathrm{IH}$, yet there were cases where the sequelae were very noticeable at the end of therapy; in these cases the use of topical $0.5 \%$ timolol gel and corticosteroids were effective for the total clearance of the IH.

The pharmacist interviewed parents at every refill visit and were able to identify potential adverse effects of treatment with propranolol, such as sleep disturbances, diarrhea, and hypotension. Adverse effects were reported in five patients, but they were of little relevance, and not a reason for discontinuation of therapy or use of an alternate treatment. Because none of the children presented with a "clinical suggestion" of hypoglycemia, no determinations were made of blood glucose in the patients during the study. Adverse effects such as bradycardia and bronchospasm were not seen in this study as reported in others [25].

Propranolol is becoming the treatment of choice in patients with $\mathrm{IH}$; but like many drugs used in pediatric patients, there are no suitable formulations available in some countries such as in México, which is why an extemporaneous formulation was used for our study. Since there are no reported clinical titration studies that correlate the dosage with the desired pharmacological effect on the type of hemangioma, the duration of treatment, reduction of the dose, and other parameters that provide an adequate treatment regimen for this group of patients, we suggest the development of such studies.

\section{Conclusions}

Treatment with propranolol administered orally at an average dose of $1.5 \mathrm{mg} / \mathrm{kg} /$ day proved to be effective in reducing the size and coloration of infantile hemangioma from the initial to the proliferative phase in Mexican pediatric patients. The administration of propranolol was safe with a minimal occurrence of adverse effects which did not interrupt the continuation of therapy.

Children starting treatment before 5 months of age show better responses and shorter duration of treatment than older children. Superficial HI had a longer duration of treatment in relation to deep and mixed ones.

Although our study only included 31 patients, we found that the duration of treatment, dose range, and presence of adverse effects can be applied in Mexican pediatric patients with this condition.

In our experience the success of therapy depended on continuous medical supervision of the patients and pharmaceutical education of the parents, which facilitated outpatient treatment and greater therapeutic adherence. The extemporaneous formulation used in this study proved to be an efficient and low-cost alternative to European formulations commercially available.

Acknowledgments The authors would like to express their thanks to the pharmacy department at Hospital Infantil de las Californias, to Dr. Victor Leon Yuri for his help in the realization of this study, and Philip O. Anderson for review of the manuscript.

\section{Compliance with Ethical Standards}

Ethical approval This study was approved by the Bioethics Committee of the Faculty of Medicine at the Autonomous University of 
Baja California in Tijuana, Mexico, with approval number 1430/2104-2. The protocol treatment was approved by the board of physicians from the Children's Hospital of the Californias.

Patient consent Written informed consent was obtained from the patients' parents for publication of this case report and any accompanying images. A copy of the written consent may be requested for review from the corresponding author.

Conflict of interest Saul Castaneda, Esbeydy Garcia, Samuel Melendez, Hermelinda de la Cruz, Oscar Ramirez, and Jose SanchezPalacio have no conflicts of interest that are directly relevant to the content of this study.

Funding No sources of funding were used to assist in the preparation of this study.

Open Access This article is distributed under the terms of the Creative Commons Attribution-NonCommercial 4.0 International License (http://creativecommons.org/licenses/by-nc/4.0/), which permits any noncommercial use, distribution, and reproduction in any medium, provided you give appropriate credit to the original author(s) and the source, provide a link to the Creative Commons license, and indicate if changes were made.

\section{References}

1. Haggstrom AN, Drolet BA, Baselga E, et al. Prospective study of infantile hemangiomas: clinical characteristics predicting complications and treatment. Pediatrics. 2006;118(3):882-7.

2. Haggstrom AN, Drolet BA, Baselga E, et al. Prospective study of infantile hemangiomas: demographic, prenatal, and perinatal characteristics. J Pediatr. 2007;150:291-1294.

3. Storch $\mathrm{CH}$, Hoeger PH. Propranolol for infantile hemangiomas: insights into the molecular mechanisms of action. Br J Dermatol. 2010;163:269-1274.

4. Itinteang T, Brasch HD, Tan ST, Day DJ. Expression of components of the renin-angiotensin system in proliferating infantile haemangioma may account for the propranolol-induced accelerated involution. J Plast Reconstr Aesthet Surg. 2011;64(6): 759-65.

5. De Lucas Laguna R. Tratamiento de los hemangiomas infantiles con propranolol. Evid Pediatr. 2011;7:79.

6. Léauté-Labrèze C, Dumas de la Roque E, Hubi-che T, Boralevi F, et al. Propranolol for severe hemangiomas of infancy. N Engl J Med. 2008;358:2649-12651.

7. Gónzalez Díaz MP, Ramos Gómez LI, Yañez Sánchez JM, Marines Sánchez HM, Páez Garza JH. Uso sistémico del propranolol en el tratamiento de hemangiomas infantiles. Rev Mex Oftalmol 2011;85(4):205-1214.

8. Orozco-Covarrubias L, García-Valencia C, Sáez-de Ocariz M, Ruiz-Maldonado R. Características clínicas y demográficas en una cohorte de niños mestizos mexicanos con hemangioma infantil. Dermatol Rev Mex. 2014;58:215-1224.

9. Beth A, Peter C. Initiation and use of propranolol for infantile hemangioma: report of a consensus conference. Pediatrics. 2013;131:128-1140.

10. Sánchez-Carpintero I, et al. Propranolol en hemangiomas infantiles: eficacia clínica, riesgos y recomendaciones. Actas Dermosifiliogr. 2011;102:766-1779.

11. Berk DR, Berk EJ, Bruckner AL. A novel method for calculating the volume of hemangiomas. Pediatr Dermatol. 2011; 28(4):478-82.

12. Henry DW, Repta AJ, Smith FM, et al. Stability of propranolol hydrochloride suspension compounded from tablets. Am J Hosp Pharm. 1986;43(6):1492-5.

13. Ensom M, Kendrick J, Rudolph S, et al. Stability of propranolol in extemporaneously compounded suspensions. Can J Hosp Pharm. 2013;66(2):118-1124.

14. Uriarte B. Víctor, Trejo F. Sergio. Farmacología clínica. $1^{\mathrm{a}}$ ed. México. Editorial Trillas; 2003, p. 309.

15. Chen T, Eichenfield L, Fallon S. Infantile hemangiomas: an update on pathogenesis and therapy. Pediatrics. 2013;131:991106.

16. Schupp CJ, Kleber J-B, Gunther P, et al. Propranolol therapy in 55 infants with infantile hemangioma: dosage, duration, adverse effects, and outcome. Pediatr Dermatol. 2011;28:640-4.

17. Hogeling M, Adams S, Wargon O. A randomized controlled trial of propranolol for infantile hemangiomas. Pediatrics. 2011;128(2):259-1266.

18. Ma X, Zhao T, Xiao Y, et al. Preliminary experience on treatment of infantile hemangioma with low-dose propranolol in China. Eur J Pediatr. 2013;172(5):653-1659.

19. Schiestl C, Neuhaus K, Zoller S, et al. Efficacy and safety of propranolol as first-line treatment for infantile hemangiomas. Eur J Pediatr. 2011;170:493-501.

20. Martinez Roca $\mathrm{C}$, et al. Oral propranolol in the treatment of infantile hemangioma: a case series of 50 infants. Eur J Pediat Dermatol. 2014;24:86-190.

21. Price CJ, Lattouf C, Baum B, McLeod M, Schachner LA, Duarte $\mathrm{AM}$, et al. Propranolol vs corticosteroids for infantile hemangiomas: a multicenter retrospective analysis. Arch Dermatol. 2011;147:1371-11376.

22. Tan S, Itinteang T, Leadbitter P. Low-dose propranolol for infantile haemangioma. J Plast Reconstr Aesthet Surg. 2011;64:292-9.

23. Andersen I, Rechnitzer C, Charabi B. Effectiveness of propranolol for treatment of infantile haemangioma. Dan Med J. 2014;61(2):A4776.

24. El Ezzi O, Hohlfeld J, de Buys Roessingh A. Propranolol in infantile haemangioma: simplifying pretreatment monitoring. Swiss Med Wkly. 2014;144:w13943.

25. Lawley LP, Siegfried E, Todd JL. Propranolol treatment for hemangioma of infancy: risks and recommendations. Pediatr Dermatol. 2009;26(5):610-4. 